\title{
There are No Universal Rules for Induction
}

\author{
John D. Norton \\ Department of History and Philosophy of Science \\ Center for Philosophy of Science \\ University of Pittsburgh \\ For updates, see \\ http://www.pitt.edu/ jdnorton
}

Prepared for Symposium "Induction Without Rules” PSA 2008: Philosophy of Science Biennial Meeting, November 2008, Pittsburgh PA.

In a material theory of induction, inductive inferences are warranted by facts that prevail locally. This approach, it is urged, is preferable to formal theories of induction in which the good inductive inferences are delineated as those conforming to some universal schema. An inductive inference problem concerning indeterministic, non-probabilistic systems in physics is posed and it is argued that Bayesians cannot responsibly analyze it, thereby demonstrating that the probability calculus is not the universal logic of induction.

\section{The Material Theory of Induction}

\subsection{What It Is}

In papers elsewhere (Norton, 2003, 2005) I have argued for a material theory of inductive inference. It urges that there are no universal rules of inductive inference; particular inductive inferences are not shown to be licit by demonstrating that they conform to one or other universally applicable schema, as is the case with deductive inference. Instead, individual inductive inferences are warranted by facts and, since the facts at issue hold contingently, the warrant inferences only in limited domains. The slogan is "all induction is local." 
The principal burden of any logic is to separate the good from the bad inferences.

Deductive logic provides the model of how a formal theory does this. Consider the deductive argument:

1. Either quantum particles in a singlet state have a non-local existence spatially or superluminal transmissions are possible.

2. Superluminal transmissions are not possible.

3. Therefore quantum particles in a singlet state have a non-local existence spatially.

Everyone sees immediately that this is a deductively valid argument. It is merely an instance of the universally applicable schema of disjunctive syllogism

\section{A or B \\ 2. Not-B \\ 3. Therefore A}

We see this without any need to grasp the content of the propositions. One may have no idea what a singlet state is or what it is for a quantum mechanical system to be non-local spatially. Nonetheless one can be assured of the validity of the argument.

Standard theories of inductive inference have long aspired to replicate this success. Maddeningly, these standard theories always seem to fall just a little short; and sometimes the shortfall is not so little. The principal difficulty is that it is impossible to judge whether an inductive inference is good without knowing what the propositions say. Here are two formally identical inductive inferences:

1. This sample of bismuth melts at $271^{\circ} \mathrm{C}$.

2. Therefore all do.

1. The first day of the new millennium was $8^{\circ} \mathrm{C}$ at noon in Pittsburgh.

2. Therefore all first days of new millennia in Pittsburgh will be so. 
Whether the inferences are good or not depends on what the propositions say - their matter or material. The first is a good inference because of the fact that elements like Bismuth are generally uniform in their properties. The second is a poor inference since we know the fact that weather is generally very variable. Indeed these facts are all we need to know to determine that the first inference is warranted and the second is not.

The natural response is that these examples are just too simple. State all the background facts properly and then the universal inductive logic that really governs the examples will come into view. This response expresses an optimistic hope that is not borne out by further efforts. For when we do try to expose this universal background logic, things become worse, not better. We may end up, for example, trying to portray the world as governed by some sort of principle of uniformity that holds for some aspects of the world but not others; and efforts to find a noncircular characterization of which are the favored aspects that enjoy uniformity become ever more cumbersome and implausible. We were better off with the simple idea that the first inference is warranted by a fact about elements and the second not warranted because the facts of weather are inhospitable.

\subsection{A Psychical Example}

The material theory urges that facts warrant inductions. Thus we should expect that two people who differ sufficiently in their assessment of the prevailing facts will also differ significantly in their judgments of the inductive import of the same evidence. This effect is illustrated in a discussion by Bertrand Russell of experimental evidence for personal survival of physical death. Russell (1957, pp. 51-52) writes

Psychical research professes to have actual scientific evidence of survival and undoubtedly its procedure is, in principle, scientifically correct. Evidence of this sort might be so overwhelming that no one with a scientific temper could reject it. The weight to be attached to the evidence, however, must depend upon the antecedent probability of the hypothesis of survival. There are always different ways of accounting for any set of phenomena, and of these we should prefer the one which is antecedently least improbable. Those who already think it likely that we survive death will be ready to view this theory as the best explanation of psychical 
phenomena. Those who, on other grounds, regard this theory as implausible will see for other explanations.

Some experimental report purports to favor survival. Those who believe that survival is possible, or those that strongly favor the survivalist thesis will infer that the experiment does inductively support a survivalist outcome. Skeptics, or those who find skepticism probable-here understood in an entirely non-technical sense as just very likely - will find a different evidential import. The facts one believes or judges probable control the inductive import each person infers.

It is hard to see in detail how this works unless we supply a more detailed illustration. Imagine that the experimental report describes a comatose person who, through an out of body experience, perceives real occurrences that are physically inaccessible. In addition, rigorous tests have established that all materialistic sensory channels (sight, sound, smell, cueing by an informer, etc.) are very unlikely to be the source of the perception. Those facts would compel us-survivalist and skeptic alike--to infer to an immaterial channel of perception through which the person can in some sense become detached from their physical body. The warrant for that inference depends entirely on the fact of the rigorous test; the extent to which one accepts that fact is the extent to which the one should find warrant for the inference.

Russell's example mentions two terms, "explanation" and "probability." They immediately suggest the two presently, most favored accounts of inductive inference. Let me explain why each is not a better explication of the example than the one offered by the material theory.

According to "inference to the best explanation" we should infer inductively to the best explanation of the evidence. Russell obligingly speaks of the "best explanation of psychical phenomena." In the more detailed example, the immaterial channel of perception would be the best explanation and we should infer to it in conformity with the principle of inference to the best explanation.

This is, in my view, a very imperfect explication of the inference. As long as we focus on the particular inference and all the pertinent facts, its compelling strength is quite evident. The moment we rise to the abstract description of it as an inference to the best explanation, its strength is obscured. We have lost essential detail. Our abstract description is merely a gloss that fails to capture what makes the specific inference successful at the strength it has. That it is the 
best explanation merely summarizes imperfectly one aspect of the inference. And that summary is rendered all the more fragile by the awkward fact that our accounts of explanation still lack the precision expected in a general theory of inference. Contrast this with the case of disjunctive syllogism above where this effect is exactly reversed. In that example, identifying the schema of disjunctive syllogism in amongst the complications of the quantum theory was all that was needed to establish the deductive force of the inference. The particular quantum mechanical assertions can be ignored; they are secondary.

When Russell urges that "we should prefer the [hypothesis] which is antecedently least improbable," Bayesians will recognize this as an idea that can be explicated in a familiar result in Bayesian epistemology. When two hypotheses entail the same evidence, the ratio of posteriors equals the ratio of priors. So we should prefer the hypothesis with the greater prior probability.

This is, once again, in my view, a very imperfect explication of the inferences at hand. The notion of "probable" and "improbable" is informal as suggested by Russell's equating of "improbable" with "implausible." One does not need to call up a mathematical theory to judge that we should prefer the antecedently most probable/plausible hypothesis if both are adequate to the evidence. We gain little from explicating these judgments in a precise mathematical calculus beyond committing the fallacy of spurious precision. Bayesians typically resist the call to assign definite numbers to the probabilities, realizing that any particular set assigned would be arbitrary and even look a little foolish. Instead they retreat to the assurance that whatever numbers they might assign will lead to pretty much the same outcome. I read that reluctance as a concession that there really is something odd and arbitrary in assigning definite numbers and that the inductive logic they have in mind is actually a much weaker logic that permits rankings only. (I suggest that Bayesians would do well to find it. See Norton, 2007.)

Bayesians will also see in Russell's remarks a suggestion of a possible Bayesian explication. Should we allow that the best explanation is the antecedently most probable of the candidate hypotheses, so that explaining better is expressed in the analysis by the assigning of a greater prior? That proposal fails because explanation and explanatory strength is a relation between the hypothesis and the thing explained, whereas a prior probability is assigned to an hypothesis only. It is easy to find hypotheses that are very good explanations of some things and explanatory failures when it comes to others. Einstein's celebrated light quantum hypothesis of 1905 affords a wonderful explanation of the photoelectric effect-so much so that it is 
mentioned in his Nobel prize award. Yet that same hypotheses is unable to explain the familiar interference effects of 19 th century optics. A single prior probability cannot simultaneous reward and punish the hypothesis.

\section{A Challenge to Bayesians}

Here I present a challenge to anyone who thinks that the probability calculus is adequate as the universal logic of induction. It is a problem in inductive inference that I do not believe Bayesians can solve responsibly.

\subsection{Indeterministic Systems in Physics}

Indeterministic systems are those for which a full specification of their present state fails to fix their future states. We have long been familiar with one manifestation of indeterminism. In quantum theory a specification of the present state of the system generally only fixes the probabilities of different futures. In a more extreme form of indeterminism, the full specification of the present leaves the future undetermined and-the key fact of importance here-our physical theories provide no physical chances for the different futures. They tell us only which futures are possible. Some recent examples arise in the supertask literature. See, for example, Alper at al. (2000); Norton, (1999). 1

One of the simplest examples in Newtonian physics is "the dome." (See Norton, 2003a, $\S 3$, manuscript.) A point mass can slide frictionlessly over a dome with circular symmetry in a vertical gravitational field. Initially, the mass is motionless at the apex. See Figure 1. If the shape of the surface is chosen appropriately, Newton's equations admit many solutions. The mass may remain at rest indefinitely at the apex; or it may remain at rest for some arbitrary time $\mathrm{T}$ and then

\footnotetext{
1 Nothing in my argument requires the reader to adopt a strong view over determinism or indeterminism in physics. All it needs the reader to accept is that some scenario with nonprobabilistic indeterminism is at least conceivable and that we would expect inductive inference to be applicable in that scenario.
} 
spontaneously accelerate in any radial direction. ${ }^{2}$ The spontaneous motion does not arise from some very slight perturbation, a miniscule wobble, say, that shakes the mass free at the moment of spontaneous excitation, time $\mathrm{t}=\mathrm{T}$. Nothing changes in conditions of the dome. It is just that Newton's equations of motion admit multiple solutions, one in which the mass remains at rest at times $\mathrm{t}>\mathrm{T}$ and one in which it moves for $\mathrm{t}>\mathrm{T}$.

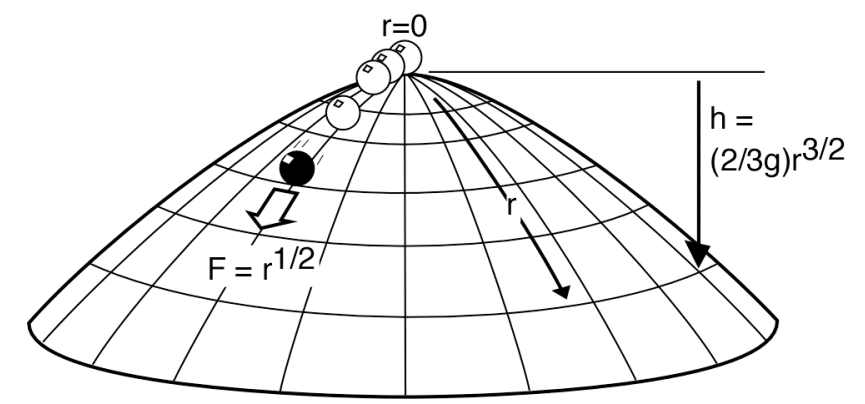

Figure 1. The dome: an indeterministic system ${ }^{3}$

A second example illustrates another sort of spontaneous excitation. An infinite sequence of identical masses and springs are laid out as mass-spring-mass-spring-... as shown in Figure 2, where the springs are governed by Hooke's law.

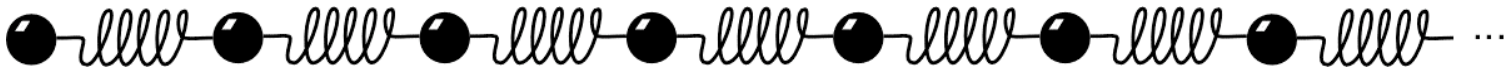

Figure 2. Masses and Springs

If the masses are initially at rest and located so that the springs are not extended or compressed, then one possible future for the system is that it remains indefinitely quiescent. There is a second

2 This happens if the surface is such that $\mathrm{h}=(2 / 3 \mathrm{~g}) \mathrm{r}^{3 / 2}$, where $\mathrm{r}$ is the radial distance in the surface of the dome and $\mathrm{h}$ the vertical distance below the apex; $\mathrm{g}$ is the acceleration due to gravity. For a unit mass on the surface, Newton's laws entail an outwardly directed acceleration field in the surface, $F=d(g h) / d r=r^{1 / 2}=d^{2} r / d^{2}$. This equation is solved by $r(t)=0$, for all $t$; and by a spontaneous excitation at $\mathrm{T}: \mathrm{r}(\mathrm{t})=0$, for $\mathrm{t} \leq \mathrm{T}$ and $\mathrm{r}(\mathrm{t})=(1 / 144)(\mathrm{t}-\mathrm{T})^{4}$, for $\mathrm{t} \geq \mathrm{T}$.

3 Figure from Norton (2003a, §3). 
possibility. The system can become spontaneously excited at some time $\mathrm{T}>0$. The mechanism is this. The first mass in the chain is accelerated after $\mathrm{T}=0$ because of a faster acceleration of the second mass; and the second mass is accelerated after $\mathrm{T}=0$ because of a faster acceleration of the third mass; and so on indefinitely. The spontaneous excitation depends essentially on the infinity of the masses; for, were there only n masses, there would be no additional $(n+1)$ th mass to excite the nth mass and initiate the process. The computation is given in Norton $(1999,1269-71) .{ }^{4}$ The full specification of the physical system merely admits this spontaneous excitation as a possibility, but supplies no probabilities for the various possible futures.

\subsection{The Inductive Inference Problem}

We are presented with an indeterministic system such as the dome or the masses and springs in a quiescent state at time $\mathrm{t}=0$. We know the full physics sketched above that governs it, so we know that spontaneous excitation is possible, and we know that no other perturbing influences will come into play. How much inductive support does this background information $\mathrm{E}$ accord to the hypothesis $\mathrm{H}\left(\mathrm{T}_{1}, \mathrm{~T}_{2}\right)$ that the system will spontaneously excite at a time $t$ in the interval $\mathrm{T}_{1} \leq \mathrm{t}<\mathrm{T}_{2}$ ?

4 The computation in Norton (1999) assumes that the mass displacements are parallel to the chain. One may wonder if that might engender collisions between the masses that might interfere with the excitation process. One escape is to presume that the masses can interpenetrate. A better one is to assume that the masses are constrained to deflections perpendicular to the chain; then may, for example, thread rigid wires perpendicular to the chain. Then curiously the formulae of the analysis of Norton (1999) remains unchanged. The displacement variable $\mathrm{x}_{\mathrm{i}}$ are now the perpendicular displacements. This comes about through the cancellation of two effects. The force exerted by a spring is reduced by the sine of angle of its direction from the chain; and the relative displacements perpendicular to the chain of the masses at the ends of that spring are reduced by this same sine. 


\subsection{A Model for the Solution}

To proceed, we take as our model the standard way of handling inductive inference problems in the context of stochastic systems where we do have probabilities-physical chances - for the possible futures. According to David Lewis' (1980) "principal principle," if we are inferring inductively over systems for which physical chances are available, we should conform our degrees of belief to those physical chances. Consider an atom of a radioactive element undergoing radioactive decay. It is governed by the law that the physical probability $\mathrm{P}(\mathrm{t})$ of the atom decaying in time $t$ is

$$
\mathrm{P}(\mathrm{t})=1-\exp (-\mathrm{t} / \tau) .
$$

where the time constant $\tau$ is related to the element's half life by $t_{1 / 2}=\tau \ln 2$. So we should conform our degrees of belief to that formula. The magnitude $\mathrm{P}(\mathrm{t})$ should be our degree of belief in the atom decaying within time $t$.

This procedure seems so straightforward as to require little justification. Once we accept that the quantum theory provides the full physical description of the system, it would simply be perverse to distributed beliefs differently and insist that the evidence accords near certainty to the hypothesis that atom will decay in its half life. For to do that is to pretend to know more about the system than the physics tells us; it is to offer a corrective to the physics.

This idea that the physical facts prevailing should determine how we should infer inductively is, of course, just the basic idea of the material theory of induction, of which Lewis' principal principle is a special case. To spell out its application, let us ask what support the evidence $\mathrm{E}$ that the atom is undecayed at time $\mathrm{t}=0$ gives to the hypothesis $\mathrm{H}(\mathrm{T})$ that the atom decays over the following time $0 \leq \mathrm{t}<\mathrm{T}$. Henceforth, let us write that degree of support as

$$
[\mathrm{H}(0, \mathrm{~T}) \mid \mathrm{E}]
$$

Since the process is fully governed by the law of radioactive decay (1), the material theory of induction enjoins us to set the degree of support equal to physical probability of decay

$$
[\mathrm{H}(0, \mathrm{~T}) \mid \mathrm{E}]=\mathrm{P}(\mathrm{T})=(1-\exp (-\mathrm{T} / \tau))
$$

What of our case in which no physical chances are to be had? The system is indeterministic, but the laws governing the multiplicity of possible futures are non-probabilistic and assign no physical chances to different possible futures. The essential insight that lies behind Lewis' "principal principle" still applies. If the realm of possibility is fully governed by some natural law, we should allow that law to dictate the course of our inductive inferences. 


\subsection{How NOT to Infer Inductively about Indeterministic Systems}

Now consider the inductive inference problem posed in Section 2.2. We have

$\mathrm{E}$ : At $\mathrm{t}=0$, the system is quiescent.

$\mathrm{H}\left(\mathrm{T}_{1}, \mathrm{~T}_{2}\right)$ : The system begins to excite at the time $\mathrm{t}$ in the interval $\left[\mathrm{T}_{1}, \mathrm{~T}_{2}\right)$, that is, in $\mathrm{T}_{1} \leq \mathrm{t}<\mathrm{T}_{2}$ ?

What is the degree of inductive support $\left[\mathrm{H}\left(\mathrm{T}_{1}, \mathrm{~T}_{2}\right) \mid \mathrm{E}\right]$ accorded by the evidence $\mathrm{E}$ to the hypothesis $\mathrm{H}\left(\mathrm{T}_{1}, \mathrm{~T}_{2}\right)$ ?

Many find in the analysis of the radioactive decay of an atom a template that they cannot resist applying to the dome. They propose

$$
[\mathrm{H}(0, \mathrm{~T}) \mid \mathrm{E}]=\mathrm{P}(\mathrm{T})=(1-\exp (-\mathrm{T} / \tau))
$$

The motivation is that the law of radioactive decay has an important property. It is the unique decay law that has "no memory." If the atom has not decayed after 1 time unit, or 5 time units, or 100 time units, then the probability of decay in the next unit of time is still the same. Speaking metaphorically, it is as if the atom does not remember how long it has survived without decay, when it decides whether to decay in each new unit of time. ${ }^{5}$

A distinctive feature of the dome and masses and springs is that they also have this "no memory" property. Whether the spontaneous excitation happens at some moment is quite independent of how long the system has been quiescent. So, if any probabilistic law is applicable to these systems, it is this one. However we cannot set our degrees of support [HIE] equal to probabilities governed by the same formula as in the law of radioactive decay. Any instance of the law of radioactive decay has a time constant $\tau$ in it. That time constant exercises a powerful influence on the chances of the spontaneous excitation. Figures 3, 4 and 5 display graphs of $\mathrm{P}(\mathrm{t})=\mathrm{P}(\mathrm{H}(0, \mathrm{t}) \mid \mathrm{E})=\mathrm{P}(\mathrm{HIE})$ for values of $\tau=0.1, \tau=1$ and $\tau=10$ :

5 The property is seen most easily by considering $\mathrm{Q}(\mathrm{t})=1-\mathrm{P}(\mathrm{t})=\exp (-\mathrm{t} / \tau)$, the probability of $n o$ decay in an initial time $t$. The probability of no decay in time $u$ subsequent to a period $t$ of no decay is just

$$
\mathrm{Q}(\mathrm{t}+\mathrm{u}) / \mathrm{Q}(\mathrm{t})=\exp (-(\mathrm{t}+\mathrm{u}) / \tau) / \exp (-\mathrm{t} / \tau)=\exp (-\mathrm{u} / \tau)=\mathrm{Q}(\mathrm{u}),
$$

which is just the probability of no decay in an initial time u. 


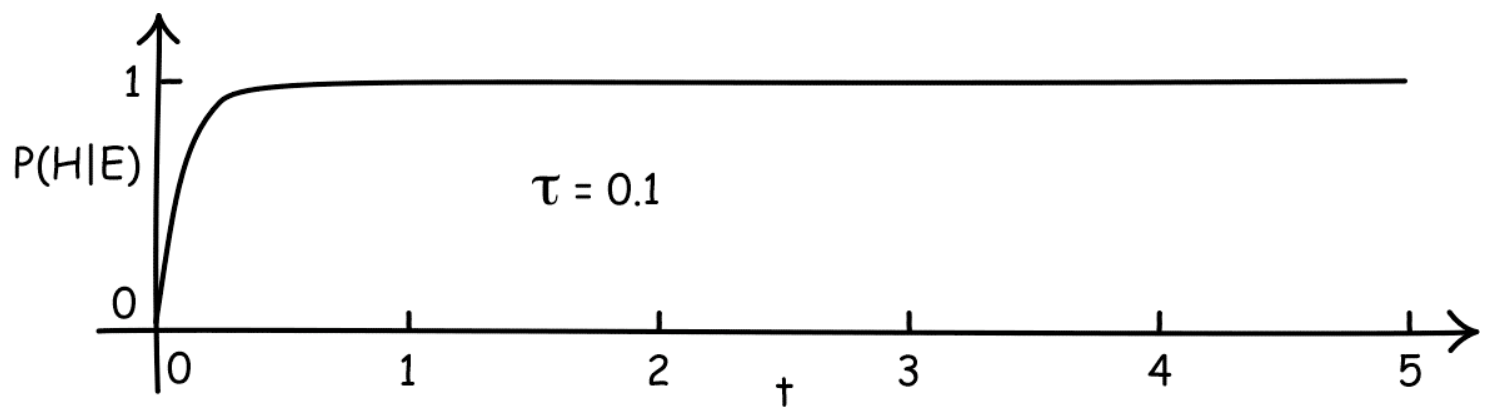

Figure 3. Decay with time constant 0.1

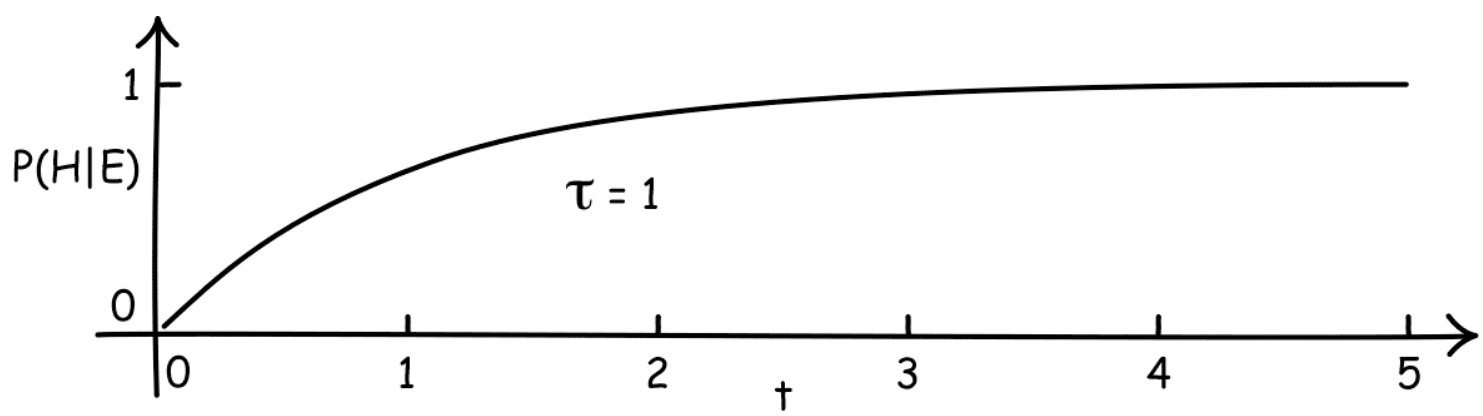

Figure 4. Decay with time constant 1

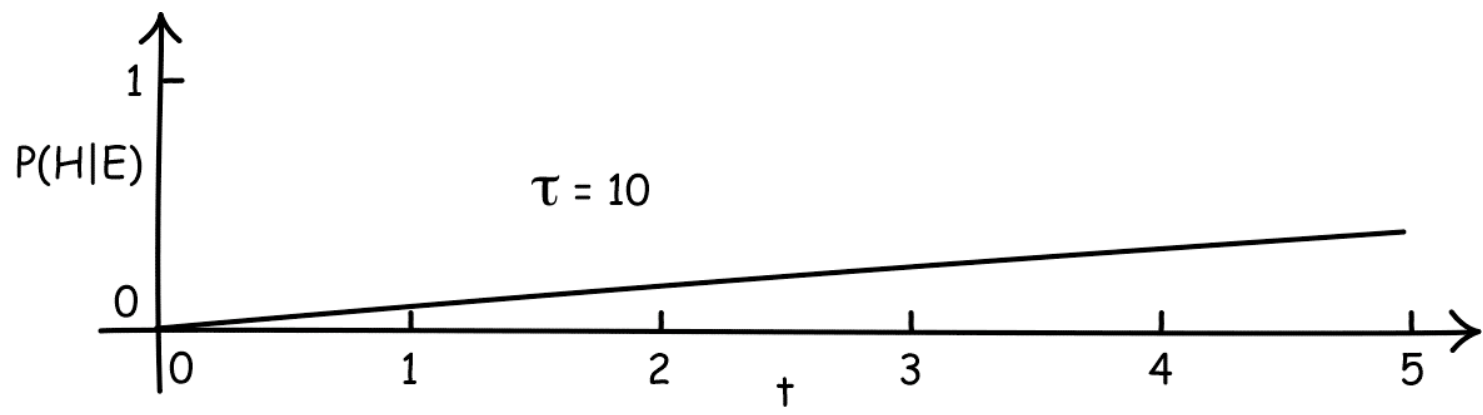

Figure 5. Decay with time constant 10

A very small time constant makes the excitation very probable, virtually immediately; a very large time constant delays the excitation very probably, for a long time. ${ }^{6}$ Nothing in the physics of the dome or masses and springs fixes a time constant or any sort of time scale for the spontaneous excitation. The physics is completely silent on how soon the motion may happen. It just says "it's possible."

6 Setting $\tau=\infty$ gives us the case of $P(T)=0$ for all $T$, so that the mass never moves. That is we assign unit probability to the outcome of no motion ever. 
So if we are to use the probabilistic formula, we must add a time scale. That is, we must pretend to know more than the full physical specification of the problem allows. Speaking metaphorically, Nature, in the guise of Newton's physics, is unable to assign a time scale to the decay. If we assign one, we must pretend to know more than Nature. Our original goal was merely to reason inductively about a system. Yet we have ended up as physicists, proposing new physical properties that the system--by construction--does not have. ${ }^{7}$

There is a loophole. Statisticians sometimes use improper probability distributions - that is, ones that do not normalize to unity - and there is an improper density with the "no memory" property. It is the uniform density $\mathrm{p}(\mathrm{H}(0, \mathrm{t}) \mid \mathrm{E})=\mathrm{dP}(\mathrm{t}) / \mathrm{dt}$, shown in Figure 5, that assigns that the same small probability $\varepsilon$ to each unit time interval.

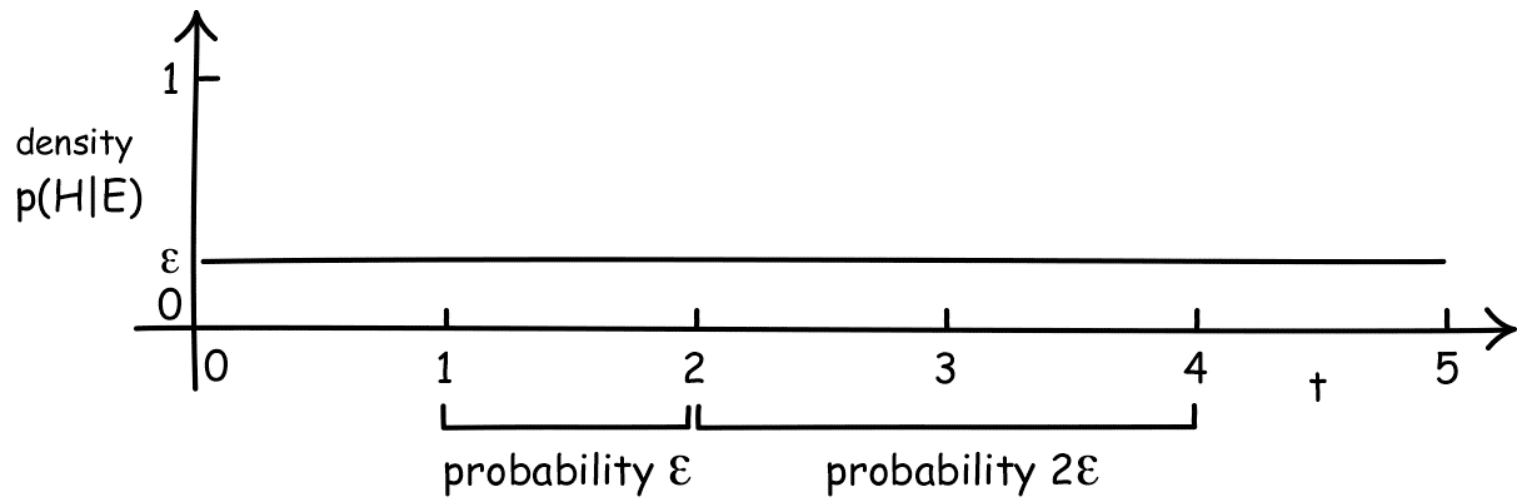

Figure 5. An Improper Probability Distribution

It is "improper" since the probability assigned to all the unit time intervals taken together does not sum to unity, as the probability calculus demands, but it is infinite.

Tempting as this improper distribution may be, it suffers the same problem as the proper distribution. It still adds physical properties. It entails is that spontaneous motion in time $t=1$ to

${ }^{7}$ Might a probabilistic analysis be possible if only we were given a little more data, such as the results of observation of several domes over some time? This strategy only makes sense if one does not accept the initial supposition that the Newtonian analysis gives the full physics of the dome. If one accepts that it does, then no catalog of outcomes will give any new, useful information for the inference problem. The situation is analogous to someone keeping detailed records of the outcomes of a roulette wheel's spins in the hope that some dependence between successive spins will be manifested. That strategy in a casino is futile if the wheel has been properly constructed so that there is no dependence. 
$t=2$ has probability $\varepsilon$ and spontaneous motion in the time interval $t=2$ to $t=4$ is $2 \varepsilon$. Motion in the one interval is twice as probable--no more no less--than motion in the other. But nothing in the physics licenses this precise judgment. All the physics says is that motion in each interval is "possible" - and that is all. Once again, we have passed from being inductive logicians to being physicists, adding more physical properties to the system than Newton's theory has already given it.

\subsection{How to Infer Inductively about Indeterministic Systems}

Something has gone very wrong. It is that we are trying to force the wrong inductive logic onto the indeterministic systems. How can we select the right one? The material theory of induction tells us that the prevailing material facts will fix the inductive logic, just as the probabilistic law of radioactive decay led us to a probabilistic logic for the radioactive decay of an atom. The physics of the indeterministic systems is more impoverished. So we should expect a more impoverished logic.

It is a somewhat mechanical exercise to read the relevant inductive logic from the physics. For radioactive decay, the chance of decay in time $t=5 \tau$ is 0.99 ; so our degree of belief in that decay is 0.99 . However the indeterministic physics of the dome and the masses and springs does not give us real-valued degrees. It just says that a spontaneous excitation in this or that time is possible; and that it all. It provides no degrees of possibility: not $50 \%$ possible, not 95\% possible; and no comparative measures: not more possible, less possible, twice as possible. It just asserts what is possible; and by logical implication we can also know what is necessary and impossible. These three assignments, necessary, possible and impossible, become the three values of our inductive logic. The translation of the material facts in the physics to the inductive logic is illustrated in the table: 


\begin{tabular}{|l|l|}
\hline What the physics says: & What it induces in the inductive logic: \\
\hline $\begin{array}{l}\text { The present state does not fix the future } \\
\text { (indeterminism). A future state is necessary, } \\
\text { possible or impossible. }\end{array}$ & $\begin{array}{l}\text { The inductive logic for the support [A|B] of A } \\
\text { from B has three values: nec, poss, imp. }\end{array}$ \\
\hline $\begin{array}{l}\text { If the excitation happens in time }[10,20), \text { then } \\
\text { it } \text { necessarily happens in }[0,100) .\end{array}$ & {$\left[\begin{array}{l}\mathrm{H}(0,100) \mid \mathrm{H}(10,20)]=\text { nec } \\
\hline \begin{array}{l}\text { Excitation in any later non-zero interval is } \\
\text { possible, given } \\
\text { E: the system is quiescent at } \mathrm{t}=0 .\end{array}\end{array}\right.$} \\
\hline $\begin{array}{l}\text { If the excitation happened in }[0,10), \text { it is } \\
\text { impossible in }[20,30) .\end{array}$ & $\begin{array}{l}{[\mathrm{H}(0,10) \mid \mathrm{E}]} \\
=[\mathrm{H}(0,100) \mid \mathrm{E}] \\
=[\mathrm{H}(10,20) \mid \mathrm{E}] \\
=\ldots=\text { poss }\end{array}$ \\
\hline
\end{tabular}

Table 1. Material Facts Dictate an Inductive Logic

The full logic is generated by simple rules:

The complete inductive logic of the indeterministic systems is ${ }^{8}$

$$
\begin{aligned}
{[\mathrm{AlB}] } & =n e c, \text { if } \mathrm{B} \text { entails } \mathrm{A} \\
& =i m p, \text { if } \mathrm{B} \text { entails not } \mathrm{A} \\
& =\text { poss, otherwise }
\end{aligned}
$$

\subsection{The Simulation Trick}

There remains a common Bayesian rejoinder. While the strengths $[\mathrm{AIB}]$ of the inductive logic just defined are not conditional probability measures, we are just a few lines of mathematics away from conditional probability measures. Consider any probability measure at all that is adapted to the behaviors of the indeterministic systems through

$$
\begin{aligned}
\mathrm{P}(\mathrm{A} \mid \mathrm{B}) & =1, \text { if } \mathrm{B} \text { entails } \mathrm{A} \\
& =0, \text { if } \mathrm{B} \text { entails not } \mathrm{A}
\end{aligned}
$$

and

$$
0<\mathrm{P}(\mathrm{A} \mid \mathrm{B})<1 \text { otherwise. }
$$

\footnotetext{
8 This logic turns out to coincide with the distribution of complete ignorance as developed in Norton (2008).
} 
Every so adapted probability measure induces the logic of Section of 2.5 by means of the formulae

$$
\begin{aligned}
{[\mathrm{A} \mid \mathrm{B}] } & =n e c, \text { if } \mathrm{P}(\mathrm{A} \mid \mathrm{B})=1 \\
& =i m p, \text { if } \mathrm{P}(\mathrm{A} \mid \mathrm{B})=0 \\
& =\text { poss, if } 0<\mathrm{P}(\mathrm{A} \mid \mathrm{B})<1
\end{aligned}
$$

Obviously there are other ways to define the logic of Section 2.5 in terms of probability measures; finding them is simply a challenge to our ingenuity.

Has this sort of possibility shown us that the probability calculus is the One True and Universal Inductive Logic after all? It has certainly not. The inductive logic of indeterministic systems are inherently non-additive. The degree of belief assigned to each of two mutually exclusive, contingent propositions is, in general, the same as the degree of belief assigned to their disjunction. If probability measures are to have any meaning as a logic of induction at all, their additivity is their essence. We add the numerical probabilities of two mutually exclusive outcomes to find the probabilities of their disjunction.

What the exercise above shows is that we can take one sort of inductive logic, a logic of additive measures, and use it to simulate another, the non-additive degrees of the logic of Section 2.5. Since the additive measures of probability theory are now offered as devices for generating all other logics, they have ceased to be used a logic in their own right. They have been reduced to a useful adjunct tool of computation and are no more the Universal Inductive Logic than is the differential calculus.

Of course we can readily simulate the additive measures of probability theory by other adjunct tools. A trivial example is provided by complex valued, multiplicative measures $\mathrm{M}$, for which we replace the additivity axiom of probability by a multiplication axiom: for mutually exclusive outcomes $\mathrm{A}$ and $\mathrm{B}, \mathrm{M}(\mathrm{AvB})=\mathrm{M}(\mathrm{A}) \cdot \mathrm{M}(\mathrm{B})$. These multiplicative measures can simulate additive measures through the formula $\mathrm{P}(\mathrm{A})=\log \operatorname{Re}(\mathrm{M}(\mathrm{A}))$, so these multiplicative measures can replicate any result achievable with additive measures. However that fact in no way makes these new measures the Universal Logic of Induction.

\section{Conclusion}

The inductive logic of indeterministic systems such as the dome and the masses and springs is non-probabilistic. For that is the sort of logic that the material facts of the system 
require. Efforts to impose a probabilistic inductive logic on these systems either require us to presume facts about the systems that outstrip their full physical description; or to reduce the probability measures to adjunct quantities in a simulation of an essentially different inductive logic.

One may doubt that the odd logic investigated has anything to do with our world, which, at least on the level of everyday experience, seems free of the indeterminism exemplified by the dome and the masses and springs. ${ }^{9}$ That is not the issue. Rather it is that these are perfectly well defined scenarios in which our ordinary modes of inference ought to be applicable. We have no hesitation in applying deductive inference to these indeterministic systems. Our deductive logics have universal scope and we fully expect the law of excluded middle to apply. If the probability calculus is the universal logic of induction, then we should expect it to apply to these indeterministic systems as well. It does not, therefore the probability calculus is not the universal logic of induction.

There are systems for which the probability calculus provides a serviceable logic of induction; and there are systems for which it does not. As we proceed away from systems for which the probability calculus provides the appropriate inductive logic, we pass to systems which require different logics. The boundary may be sharp or it may be less well defined. The pressing problem for an inductive logician is to determine where this boundary lies and to be able to decide which side to place each new problem in inductive inference as it arises. The material theory of induction provides a direct solution to the problem: the facts prevailing in the domain of the problem fix the applicable inductive logic.

\section{References}

Alper, Joseph S., Bridger, Mark, Earman, John and Norton, John D. (2000) "What is a Newtonian System? The Failure of Energy Conservation and Determinism in Supertasks," Synthese, 124, pp. 281-293.

\footnotetext{
9 There may be non-probabilistic indeterminism in our world. Standard big bang cosmology allows many different matter densities to emerge from the big bang singularity without assigning probabilities to the different possibilities.
} 
Lewis, David (1980) "A Subjectivist's Guide to Objective Chance," pp. 263-93 in R.C. Jeffrey ed., Studies in Inductive Logic and Probability. Berkeley: University of California Press.

Norton, John D. (2003) “A Material Theory of Induction," Philosophy of Science, 70, pp. 64770.

Norton, John D. (2003a) “Causation as Folk Science,” Philosophers' Imprint, 3(No.4); www.philosophersimprint.org/003004/; reprinted as Ch. 2 in, H. Price and R. Corry, eds., Causation, Physics and the Constitution of Reality. Oxford University Press, 2007.

Norton, John D. (2005) "A Little Survey of Induction," in P. Achinstein, ed., Scientific Evidence: Philosophical Theories and Applications. Johns Hopkins University Press. pp. 9-34.

Norton, John D. (2007) "Probability Disassembled" British Journal for the Philosophy of Science, 58, pp. 141-171.

Norton, John D. (2008) "Ignorance and Indifference." Philosophy of Science. Scheduled for January 2008 issue.

Norton, John D. (manuscript),"The Dome: An Unexpectedly Simple Failure of Determinism," PSA 2006: Proceedings of the 2006 Biennial Meeting of the Philosophy of Science Association, Philosophy of Science, forthcoming.

Russell, Bertrand (1957) Why I am not a Christian. George Allen and Unwin; repr. Touchstone. 